\title{
QUANTIFICATION OF ACUTE MYOCARDIAL DAMAGE SECONDARY TO IMPLANTATION OF ELECTRODES FOR THE LEFT BUNDLE BRANCH AREA PACING
}

\author{
Yucheng Wu, Yuanyuan Chen, Meng Chen, Qin Chang, Zhixin Jiang, Xiujuan Zhou, and Qijun Shan* \\ Department of Cardiology, The First Affiliated Hospital of Nanjing Medical University, Jiangsu Province Hospital, \\ Jiangsu, China
}

\begin{abstract}
Background: Different from the traditional right ventricular pacing, the left bundle branch area pacing (LBBAP) is accomplished with deeper lead implantation and more attempts. However, myocardial damage is unclear in LBBAP. Objective: The objective of the study was to observe the change of troponin $T$ and explore possible factors associated with greater myocardial damage in LBBAP. Methods: Patients with an indication for pacemaker implantation underwent attempts for LBBAP by transventricular septal method. Levels of troponin T were determined before operation, $12 \mathrm{~h}$ and 1 week after the operation. Parameters of intraoperation and follow-up were recorded and analyzed. Results: In total, successful LBBAP was achieved in 126 patients. The levels of troponin $\mathrm{T}$ increased significantly at $12 \mathrm{~h}$ after the operation compared with those before operation ( $96.45 \pm 11.07$ [69.06] vs. $16.59 \pm 1.84$ [11.92] ng/L, p < 0.001), while there were no significant differences between pre- and post-operative levels at 1 week. Correlation and regression analysis showed that only the number of attempts was an independent factor related to the change of troponin T. During 1 year of follow-up, LBBAP was safe and feasible with few complications. Conclusions: Myocardial damage of LBBAP was clinically significant. The number of attempts was an independent factor related to the myocardial damage. (REV INVEST CLIN. 2021;73(3):XX-XX)
\end{abstract}

Key words: Left bundle branch area pacing. Myocardial damage. Troponin T.

\section{INTRODUCTION}

Permanent pacemaker implantation is accomplished by transvenous insertion of endocardial leads. A number of studies have demonstrated that the mechanical effect of lead fixation on the myocardium may cause local injury, as evidenced by raised levels of plasma troponin and inflammatory biomarkers ${ }^{1,2}$. It is reported that transvenous insertion of the leads is followed by a sequence of cardiac histopathological changes, leading eventually to the formation of a fibrous connective tissue scar ${ }^{3}$. In the traditional way of pacing, most patients have a very slight troponin elevation after pacemaker implantation, leading to consider that it is not harmful to cardiac function and structure ${ }^{4}$.

Physiological pacing has always been the goal that clinicians have been striving for. From the recording of
*Corresponding author:

Qijun Shan

E-mail: qjshan@njmu.edu.cn
Received for publication: 05-09-2020

Approved for publication: 10-11-2020

DOI: $10.24875 / R I C .20000457$

0034-8376 / (c) 2020 Revista de Investigación Clínica. Published by Permanyer. This is an open access article under the CC BY-NC-ND license (http://creativecommons.org/licenses/by-nc-nd/4.0/). 
His bundle electrogram ${ }^{5}$ to the success of His bundle pacing $(\mathrm{HBP})^{6}$, physiological pacing has made significant progress. However, high pacing thresholds and low R-wave amplitudes limited the clinical application of $\mathrm{HBP}^{7}$. As the most potentially physiological pacing method, the left bundle branch area pacing (LBBAP) has been proved feasible, with stable parameters and many benefits ${ }^{8,9}$. Different from the traditional right ventricular pacing (RVP), the ventricular lead implantation in LBBAP is accomplished by the transventricular septal method. In addition, the final electrode implantation depth is 7-11 mm, which is much deeper than that of traditional pacing ${ }^{10}$. Moreover, LBBAP is not guaranteed to be in place on the first attempt, and it may take several attempts to succeed.

It is unclear what is the myocardial damage of deeper lead implantation and of more attempts in LBBAP. The severity of myocardial damage in LBBAP needs to be evaluated with clinical data. Therefore, this study aimed to observe the changes of troponin $T$ in patients undergoing LBBAP and explore possible factors associated with greater myocardial damage in LBBAP.

\section{METHODS}

\section{Patient selection}

From March 2019 to December 2019, patients who had symptomatic bradycardia with an indication for pacemaker implantation according to 2013 ESC/ EHRA Guidelines ${ }^{11}$ underwent attempts for LBBAP at Jiangsu Province People Hospital after informed written consent was obtained. Patients were excluded if they underwent cardiac resynchronization therapy or implantable cardioverter defibrillator implantation. This study was approved by the hospital ethics committee.

\section{LBBAP procedure}

LBBAP was achieved by the transventricular septal method in the basal ventricular septum and performed using the Select Secure pacing lead (Model $383069 \mathrm{~cm}$, Medtronic Inc., Minneapolis, MN, USA), delivered through a fixed curve sheath (C315 HIS, Medtronic Inc., Minneapolis, MN, USA) ${ }^{12}$. During implantation, the tip electrode of the lead was used for unipolar pacing and recording. Briefly, the delivery sheath with the lead tip just beyond the distal part of the sheath under anteroposterior position or $30^{\circ}$ right anterior oblique (RAO) was first inserted into the His bundle region where the His bundle potential (HBP) was recorded. The His bundle region was referred as a marker; the initial site for LBBAP is approximately $1-1.5 \mathrm{~cm}$ distal to the HBP lead position in the right ventricular (RV) septum along the line between the HBP site and RV apex under $30-^{\circ}$ RAO fluoroscopic view. Once the lead touched the right side of the septum and paced QRS morphology showed a "w" pattern with a notch at the nadir of the QRS in lead V1 during pacing with an output of 5 $\mathrm{V} / 0.5 \mathrm{~ms}$, the lead was pointed towards the left side of the septum and screwed in place. During the lead advancement procedure, paced QRS morphology and pacing impedance were closely monitored. Once paced QRS morphology showed right bundle branch block (RBBD) (usually Qr or rSR' in electrocardiogram [ECG] lead V1) or near-normal QRS complex, the lead advancement was stopped. The depth of the lead inside the ventricular septum could be estimated by fluoroscopic imaging with contrast injection. Successful LBBAP was defined as unipolar paced QRS with RBBD morphology and QRS duration (QRSd) $<130$ $\mathrm{ms}^{13}$. If an acceptable LBBAP could not be achieved after attempts at five locations, then the lead was placed in the left ventricular septum ${ }^{14}$.

\section{Measurements}

The levels of troponin T were recorded before operation, $12 \mathrm{~h}$ after operation, and 1 week after operation. Intraoperative parameters were recorded as follows: number of attempts, number of angiographies, time of 3830 lead implantation, radiation dose of $\mathbf{3 8 3 0}$ lead implantation, depth of 3830 lead implantation, and initial interventricular septal thickness. Pacing threshold, R-wave amplitude, and impedance were recorded during operation and at 1 week and 1 year after operation. Echocardiogram and ECG of patients were obtained at 1 week and 1 year of the operation.

\section{Statistics}

Categorical data were presented as numbers (\%), and continuous variables with normal distribution were reported as mean \pm standard error (Mean \pm SE). Mean supplemented by SE and median (Mean \pm SE [median]) were presented for skewed distribution 
continuous variables. Because the assumption of normal distribution was violated for troponin $T$ levels (Kolmogorov-Smirnov test), non-parametric Kruskal-Wallis test was performed. Pearson's correlation analysis was used to examine the relationship between levels of troponin $T$ and possibly related factors. Multiple linear regression analysis was used to explore possible factors associated with greater myocardial damage. A two-sided $p<0.05$ was considered statistically significant. All analyses were performed using SPSS statistical software (version 18; SPSS, Chicago, IL).

\section{RESULTS}

\section{Patients}

In total, 138 patients who underwent attempted LBBAP for symptomatic bradycardia were enrolled in this study. Finally, LBBAP was successfully achieved in 126 patients; in the remaining 12 patients, it failed and left ventricular septum pacing was chosen. Clinical characteristics of patients with LBBAP are summarized in table 1 . The indications of pacemaker implantation were sinus node dysfunction in 70 patients (56\%), atrioventricular block in 48 patients (38.4\%), and atrial fibrillation with long RR interval in 7 patients (5.6\%). Figure 1 shows the position of 3830 leads in the interventricular septum in successful LBBAP by $\mathrm{X}$-ray and echocardiogram. Figure 2 shows the developing process of pacing ECG during LBBAP and the fluoroscopic images of sheath angiography and final stage.

\section{Change of troponin $\mathrm{T}$}

Figure 3 shows the changes of troponin T levels in patients undergoing LBBAP before operation, $12 \mathrm{~h}$ after operation, and 1 week after operation, with statistical differences. Compared with those before the operation, the levels of troponin $\mathrm{T}$ increased significantly at $12 \mathrm{~h}$ after operation $(96.45 \pm 11.07$ [69.06] vs. $16.59 \pm 1.84$ [11.92] ng/L, p $<0.001$ ). The levels of troponin $\mathrm{T}$ were significantly decreased at 1 week after operation compared with $12 \mathrm{~h}$ after operation (31.16 \pm 4.04 [19.73] vs. $96.45 \pm 11.07$ [69.06] ng/L, p < 0.001), and there were no significant differences compared with levels before the operation.
Table 1. Clinical characteristics of patients with successful LBBAP

\begin{tabular}{lc}
\hline & All \\
\hline $\mathrm{N}$ & 126 \\
Age, $\mathrm{y}$ & $70.4 \pm 1.3$ \\
Male, $\mathrm{n}$ & 68 \\
CAD, $\mathrm{n}$ & 26 \\
Hypertension, $\mathrm{n}$ & 72 \\
DM, n & 16 \\
LVEF, \% & $62.0 \pm 0.6$ \\
IVS, mm & $10.3 \pm 0.2$ \\
Baseline QRSd, ms & $95.5 \pm 2.9$ \\
SND, $\mathrm{n}$ & 70 \\
AVB, $\mathrm{n}$ & 49 \\
AF with long RR interval & 7 \\
\hline
\end{tabular}

Categorical data are presented as number, and continuous data are presented as mean $\pm \mathrm{SE}$.

CAD: coronary artery disease; DM: diabetes mellitus;

LVEF: left ventricular ejection fraction; IVS: interventricular septum; QRSd: QRS duration; SND: sinus node dysfunction;

AVB: atrioventricular block; AF: atrial fibrillation.

\section{Correlation and regression analyses}

Pearson's correlation analysis showed that the increase of troponin $T$ was positively correlated with the number of attempts, the number of angiographies, time of 3830 lead implantation, and radiation dose of 3830 lead implantation, but not correlated with the depth of electrode implantation and the thickness of the interventricular septum. Multiple linear regression analysis found that only the number of attempts was an independent factor related to the change of troponin $\mathrm{T}$ levels (Table 2).

\section{Safety of model 3830 lead implantation}

Five patients ( $4.0 \%$ ) had model 3830 lead perforation (electrodes penetrating through the interventricular septum into the left ventricle), and 2 patients ( $1.6 \%$ ) had electrode dislocation (electrodes falling out of the fixed position) during the procedure. On examination, these patients did not show symptoms or signs of discomfort. At 1 week and at 1 year after the operation, no more lead perforations and dislocations occurred. Five patients (4.0\%) at 1 week and 10 patients $(7.9 \%)$ at 1 year after the operation had worsened tricuspid regurgitation from normal to mild, 
Figure 1. Position of the 3830 lead in the interventricular septum. A, Final fluoroscopic image of the 3830 lead. B, Final echocardiogram image of the 3830 lead. The white arrows refer to the head part of 3830 lead stuck in the interventricular septum.

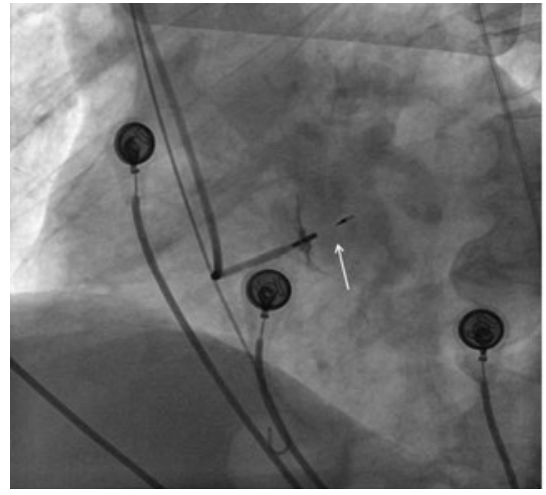

A

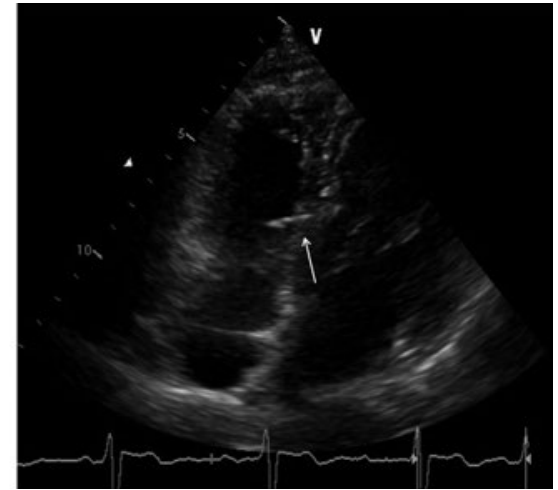

B

Figure 2. The developing process of pacing electrocardiogram and fluoroscopic image of LBBAP. A, the developing process of pacing electrocardiogram ( $3.5 \mathrm{~V}$ at $0.5 \mathrm{~ms}$ ) during LBBAP from pre-operative to final. $\mathrm{B}$, the angiography image shows the position of 3830 lead stuck in the interventricular septum. C-E, final fluoroscopic image in three projections (AP, LAO40 ${ }^{\circ}$, and RAO30 ${ }^{\circ}$ ) of LBBAP. PRE: pre-operative; LBB: left bundle branch; RA: right atrium; IVS: interventricular septum.

A

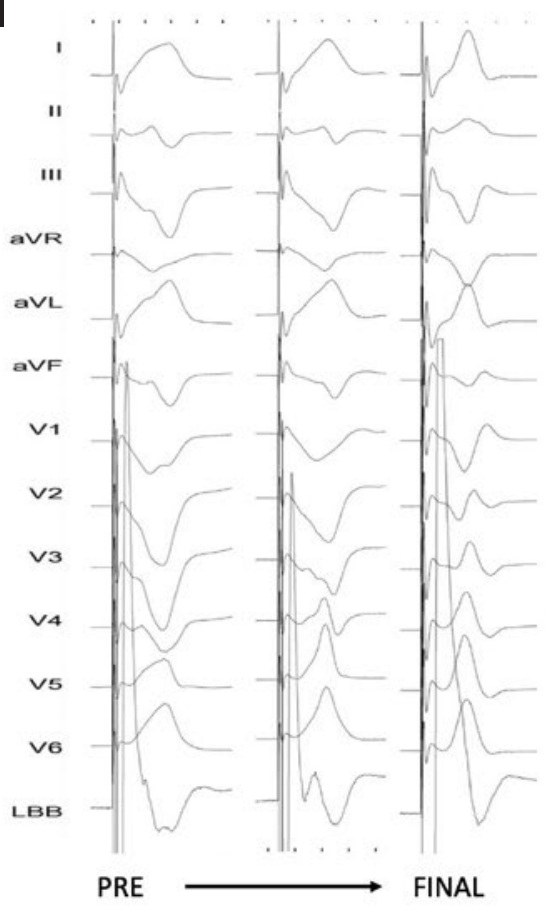

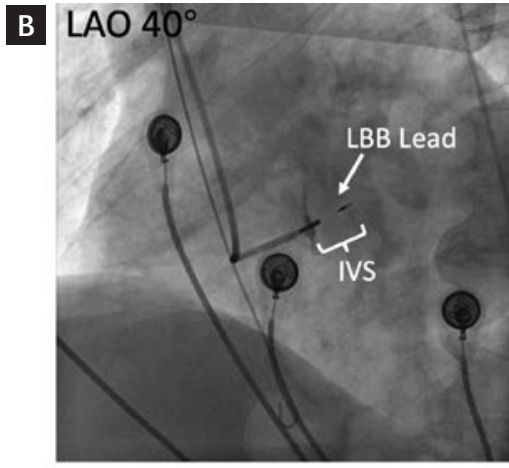

D

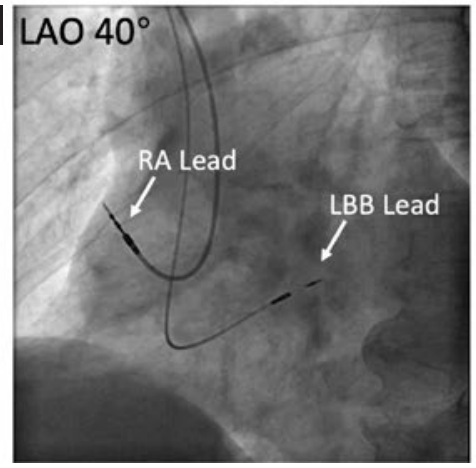

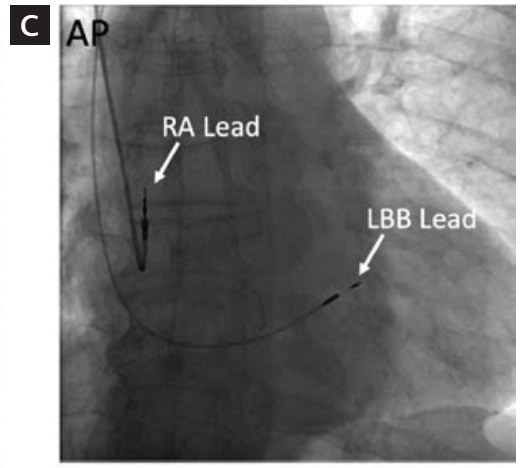

$\mathrm{E} \operatorname{RAO} 30^{\circ}$

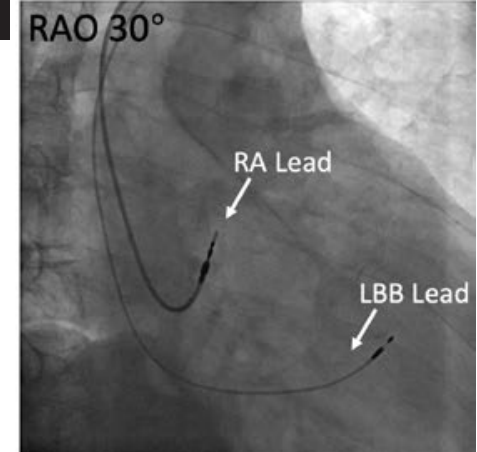

although there was no obvious restriction in leaflet motion of the lead. Threshold, R-wave amplitude, and impedance of the 3830 lead were stable and reliable during the procedure and follow-up (Table 3). Overall, the incidence of complications in this study was very low, and there were no serious complications during the 1-year follow-up.

\section{Comparison with other types of ventricular pacing}

By retrieving related reports, we compared the troponin $T$ change between RVP in a previous study ${ }^{4}$ and LBBAP in this study. As shown in table 4, the myocardial damage of LBBAP was more serious than that of 
Figure 3. Changes of troponin T levels. Troponin T pre-operative, $12 \mathrm{~h}$ post-operative, and 1 week post-operative levels in patients undergoing LBBAP were compared. Pre-op, pre-operative; $12 \mathrm{~h}$ post-op, $12 \mathrm{~h}$ post-operative; $1 \mathrm{w}$ post-op, $1 \mathrm{week}$ postoperative; cTNT, cardiac troponin T; ns, not significant; ${ }^{* * *}, \mathrm{p}<0.001$.

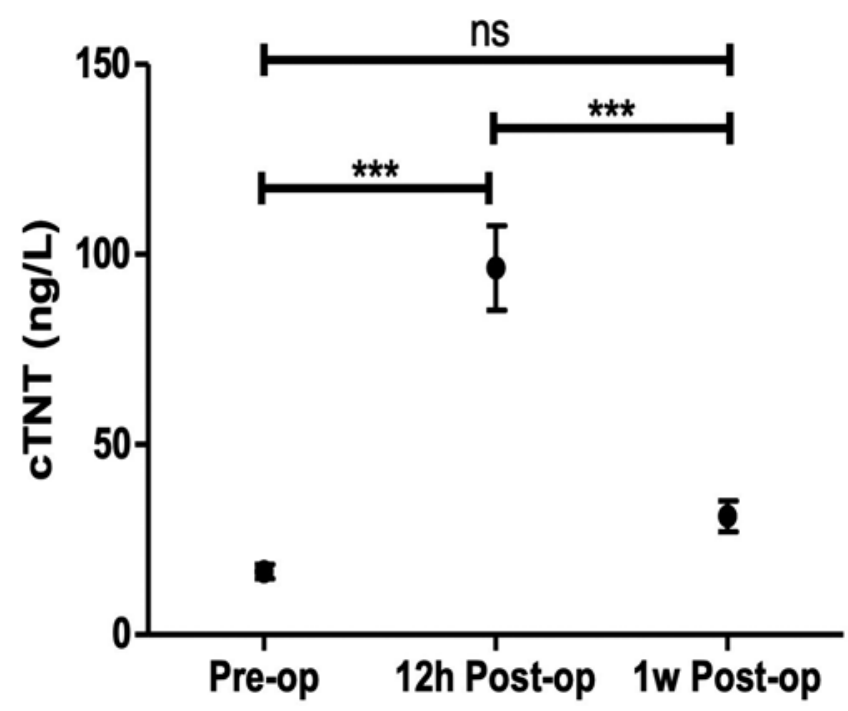

Table 2. Correlation and regression analyses

\begin{tabular}{|c|c|c|c|c|}
\hline & \multicolumn{2}{|c|}{ Correlation analysis } & \multicolumn{2}{|c|}{ Regression analysis } \\
\hline & $r$ value & $p$ value & $\begin{array}{c}\text { B } \\
(95 \% \mathrm{Cl})\end{array}$ & $p$ value \\
\hline Number of attempts & 0.548 & $0.000^{*}$ & $\begin{array}{c}18.329 \\
(12.253-24.405)\end{array}$ & $0.000^{*}$ \\
\hline Number of angiographies & 0.288 & $0.002^{*}$ & $\begin{array}{c}6.719 \\
(-14.380-27.817)\end{array}$ & 0.529 \\
\hline $\begin{array}{l}\text { Time of } 3830 \text { lead } \\
\text { implantation }\end{array}$ & 0.480 & $0.000^{*}$ & $\begin{array}{c}0.751 \\
(-0.274-1.777)\end{array}$ & 0.148 \\
\hline $\begin{array}{l}\text { Radiation dose of } 3830 \\
\text { lead implantation }\end{array}$ & 0.055 & 0.802 & - & - \\
\hline $\begin{array}{l}\text { Depth of } 3830 \text { lead } \\
\text { implantation }\end{array}$ & -0.141 & 0.239 & - & - \\
\hline $\begin{array}{l}\text { Thickness of } \\
\text { interventricular septum }\end{array}$ & 0.105 & 0.312 & - & - \\
\hline
\end{tabular}

*Statistically significant.

We explored the overall population to identify variables correlated with the increase of troponin T. Linear regression analysis was used to adjust for covariates that were found in the correlation analysis.

RVP, and the ratio of troponin T levels exceeding normal range ( $>30 \mathrm{ng} / \mathrm{L}$ ), or the range of minimal myocardial damage ( $>90 \mathrm{ng} / \mathrm{L}$ ) was both higher than that of RVP. No literature was retrieved on myocardial damage in HBP.

Figure 4 shows that in this study, a patient with a dual chamber pacemaker who previously underwent right ventricular apical pacing (RVAP) was treated by pacemaker replacement using LBBAP due to battery depletion. The original ventricular electrode was abandoned in the right ventricle without being pulled out. As shown in the figure, LBBAP, HBP, and RVAP were quite different in pacing ECG and fluoroscopic images. In the pacing ECG, LBBAP shows as an incomplete RBBD with narrow QRS, HBP as an approximately normal ECG, while RVAP as a complete left bundle branch block with wide QRS. 
Table 3. Safety of model 3830 lead implantation

\begin{tabular}{|c|c|c|c|}
\hline & Intraoperative & 1 week post-operative & 1 year post-operative \\
\hline Lead perforation ( $n$ ) & 5 & 0 & 0 \\
\hline Lead dislocation (n) & 2 & 0 & 0 \\
\hline $\begin{array}{l}\text { Worsened tricuspid } \\
\text { regurgitation }(n)\end{array}$ & - & 5 & 10 \\
\hline $\begin{array}{l}\text { Interventricular septal motion } \\
\text { abnormality }(n)\end{array}$ & 0 & 0 & 0 \\
\hline Threshold ( $v$ at $0.5 \mathrm{~ms}$ ) & $0.56 \pm 0.11$ & $0.54 \pm 0.12$ & $0.75 \pm 0.25$ \\
\hline R-wave amplitude (mv) & $12.5 \pm 2.6$ & $13.7 \pm 3.5$ & $10.3 \pm 3.7$ \\
\hline Impedance $(\Omega)$ & $772 \pm 15$ & $656 \pm 22$ & $706 \pm 36$ \\
\hline
\end{tabular}

Table 4. Comparison of troponin T change between LBBAP and RVP

\begin{tabular}{lcc}
\hline & LBBAP & RVP \\
\hline Troponin T (ng/L) & Baseline: & Baseline: \\
& $11.9(7.3-20.0)$ & $12(8-17)$ \\
& Post-operative: & Post-operative: \\
Post-operative $>30 \mathrm{ng} / \mathrm{L}$ & $69.1(37.3-143.5)$ & $32-50)$ \\
Post-operative $>90 \mathrm{ng} / \mathrm{L}$ & $84.3 \%$ & $55.6 \%$ \\
\hline
\end{tabular}

Continuous data are presented as median (25-75th percentiles). LBBAP: left bundle branch area pacing; RVP: right ventricular pacing.

\section{DISCUSSION}

This study aimed to observe myocardial damage in LBBAP, a new physiological pacing way, through analyzing the changes of troponin $T$ levels in patients undergoing LBBAP. We found that the level of troponin $\mathrm{T}$ increased significantly at $12 \mathrm{~h}$ after operation and returned to pre-operative levels in 1 week. By retrieving and comparing related reports, we found that the myocardial damage of LBBAP was more serious than that of RVP.

The results above suggest that the myocardial damage of this new pacing mode is clearly existing. The previous studies have shown that the damage caused by traditional RVP is not clinically significant ${ }^{15}$. Different from the traditional RVP, the ventricular lead of LBBAP needs to penetrate into the interventricular septum to reach the left ventricular endocardium ${ }^{16}$. The ventricular lead of LBBAP into the myocardium is much deeper than that of RVP, and it needs more attempts to succeed, which we think is the main cause of myocardial damage. However, at 1 week after operation, troponin $\mathrm{T}$ returned to the pre-operative level, and no obvious interventricular septal motion abnormality was found in the follow-up echocardiography. These results indicated that the myocardial damage was transient and recoverable, without damage to myocardial contraction.

As the myocardial damage was clear, it was important to find the related factors to reduce the damage. Then, correlation and regression analyses showed that only the number of attempts was an independent factor related to the change of troponin $\mathrm{T}$. Therefore, achieving success with the least number of attempts is an effective way to reduce myocardial damage. The previous studies have pointed out that the ventricular nine partition method ${ }^{17}$ and typical bundle branch block morphology ${ }^{18}$ are effective to predict the success of LBBAP. Besides, intracardiac echocardiogram was an efficient tool to guide LBBAP $^{19}$. The above methods or indicators increase the probability of success and decrease the number of 
Figure 4. Comparison of pacing electrocardiogram and fluoroscopic image between LBBAP, HBP, and RVAP. A, comparison of pacing electrocardiogram ( $3.5 \mathrm{~V}$ at $0.5 \mathrm{~ms}$ ) between LBBAP, HBP, and RVAP. B, fluoroscopic image of 3830 lead in His position. $\mathrm{C}-\mathrm{E}$, final fluoroscopic image in three projections (AP, $\mathrm{LAO} 40^{\circ}$, and $\mathrm{RAO} 30^{\circ}$ ) of a patient treated by pacemaker replacement from RVAP to LBBAP. LBBAP: left bundle branch area pacing; HBP: His bundle pacing; RVAP: right ventricular apical pacing; RVA: right ventricular apex; LBB: left bundle branch; RA: right atrium.
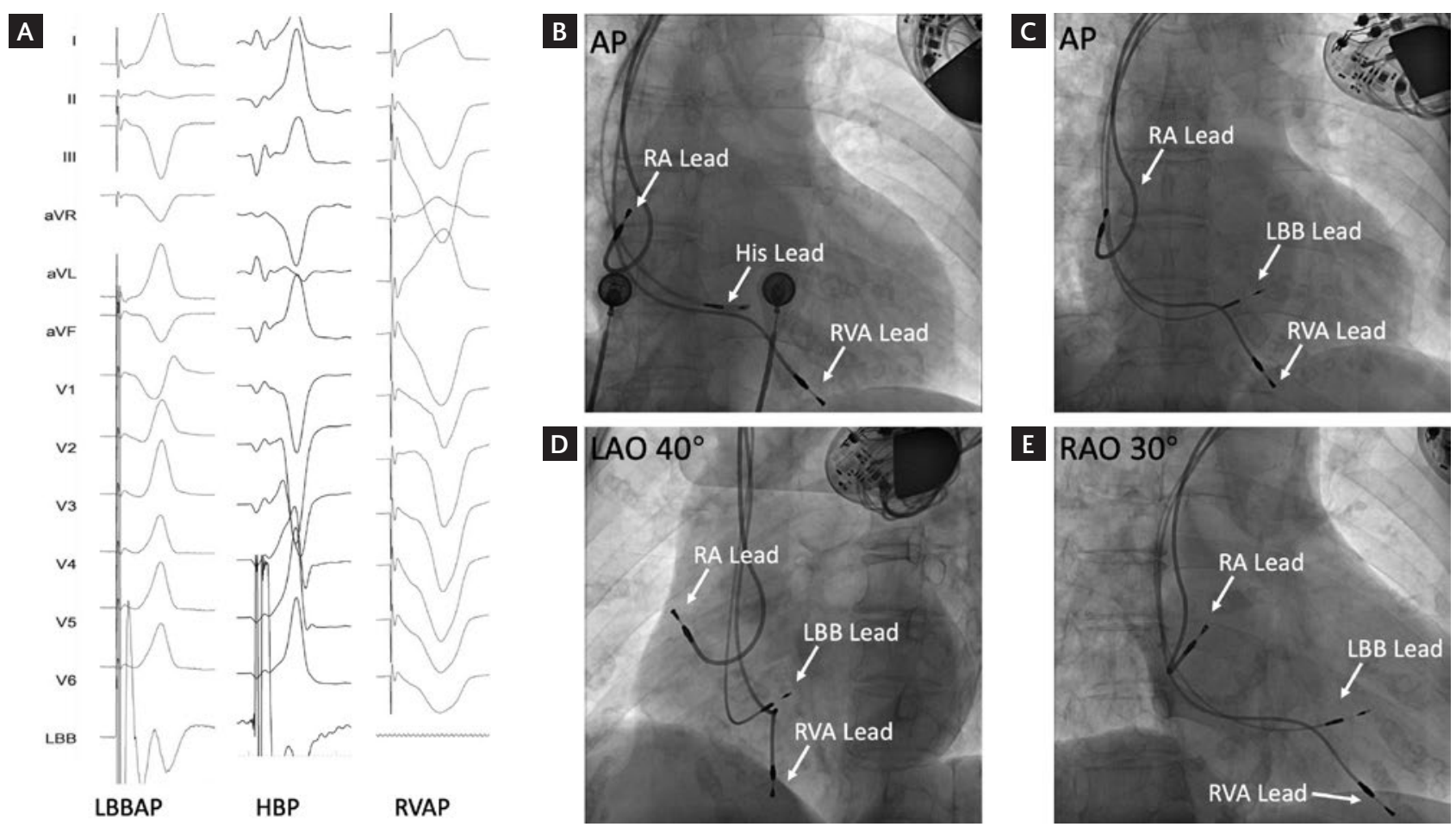

attempts, thereby reducing myocardial damage. Of course, in some cases which are difficult to correct or succeed, other alternatives such as left ventricular septum pacing should be chosen to avoid serious myocardial damage or complications caused by too many attempts ${ }^{20}$.

In terms of complications, there were no lead perforation, lead dislocation, or interventricular septal motion abnormality during a 1-year follow-up. Mild tricuspid regurgitation occurred in 10/126 patients, not more than that seen in conventional RVP21. Besides, threshold, R-wave amplitude, and impedance were stable and reliable during the procedure and follow-up.

There are some limitations in this study. First, this is a single-center study with a small sample size and without control groups. Besides, the above results did not eliminate the influence of atrial electrode implantation. Finally, longer follow-up is needed to consolidate the conclusions of this study.
In conclusion, acute myocardial damage of LBBAP was clinically significant, which seemed more serious than RVP. There were few complications during 1 year of follow-up. The number of attempts was an independent factor related to the myocardial damage.

\section{ACKNOWLEDGMENTS}

We would like to thank our colleagues, clinical engineers, and support staff for their help with this study.

\section{REFERENCES}

1. Hnatek T, Taborsky M, Maly M, Kamenik L, Littnerova S, Sedlon $P$, et al. Factors underlying elevated troponin I levels following pacemaker primo-implantation. Biomed Pap Med Fac Univ Palacky Olomouc Czech Repub. 2016;160:248-56.

2. Huang GY, Peng Z, Zhan Y, Liu DD, Liu YL. Relationship between the change in pacing threshold and the myocardial injury. Eur Rev Med Pharmacol Sci. 2017;21:5774-80.

3. Varvarousis D, Goulas N, Polytarchou K, Psychari SN, Paravolidakis $\mathrm{K}$, Konstantinidou $\mathrm{A}$, et al. Biomarkers of myocardial in- 
jury and inflammation after permanent pacemaker implantation: the lead fixation type effect. J Atr Fibrillation. 2018; 10:1798.

4. Chen X, Yu Z, Bai J, Hu S, Wang W, Qin S, et al. Troponin T elevation after permanent pacemaker implantation. J Interv Card Electrophysiol. 2017;49:211-8.

5. Márquez MF, Moukabary T, Gonzalez MD. Jesús Alanís and the first recording of the His bundle: the scientist and the man. Pacing Clin Electrophysiol. 2014;37:1724-7.

6. Vijayaraman P, Chung MK, Dandamudi G, Upadhyay GA, Krishnan K, Crossley G, et al. His bundle pacing. J Am Coll Cardiol. 2018;72:927-47

7. Sharma PS, Vijayaraman P, Ellenbogen KS. Permanent His bundle pacing: shaping the future of physiological ventricular pacing. Nat Rev Cardiol. 2020;17:22-36.

8. Li Y, Chen K, Dai Y, Li C, Sun Q, Chen R, et al. Left bundle branch pacing for symptomatic bradycardia: implant success rate, safety, and pacing characteristics. Heart Rhythm. 2019;16: $1758-65$.

9. Das A, Islam SS, Pathak SK, Majumdar I, Sharwar SA, Saha R, et al. Left bundle branch area. A new site for physiological pacing: a pilot study. Heart Vessels. 2020;35:1563-72

10. Slotwiner DJ, Raitt MH, Munoz FD, Mulpuru SK, Nasser N, Peterson PN. Impact of physiologic pacing versus right ventricular pacing among patients with left ventricular ejection fraction greater than $35 \%$ : a systematic review for the 2018 ACC/AHA/ HRS Guideline on the evaluation and management of patients with bradycardia and cardiac conduction delay: a report of the American college of cardiology/American heart association task force on clinical practice guidelines and the heart rhythm society. Circulation. 2019;140:e483-503.

11. Brignole M, Auricchio A, Baron-Esquivias G, Bordachar P, Boriani G, Breithardt OA, et al. 2013 ESC guidelines on cardiac pacing and cardiac resynchronization therapy: the task force on cardiac pacing and resynchronization therapy of the European society of cardiology (ESC). Developed in collaboration with the European heart rhythm association (EHRA). Eur Heart J. 2013; 34:2281-329.
12. Li X, Li H, Ma W, Ning X, Liang E, Pang K, et al. Permanent left bundle branch area pacing for atrioventricular block: feasibility, safety, and acute effect. Heart Rhythm. 2019;16:1766-73.

13. Chen K, Li Y, Dai Y, Sun Q, Luo B, Li C, et al. Comparison of electrocardiogram characteristics and pacing parameters between left bundle branch pacing and right ventricular pacing in patients receiving pacemaker therapy. Europace. 2019;21: 673-80.

14. Mafi-Rad M, Luermans JG, Blaauw $Y$, Janssen M, Crijns HJ, Prinzen FW, et al. Feasibility and acute hemodynamic effect of left ventricular septal pacing by transvenous approach through the interventricular septum. Circ Arrhythm Electrophysiol. 2016; 9:e003344

15. Blazek P, Ferri-Certic J, Vrazic H, Lennerz C, Grebmer C, Kaitan $\mathrm{K}$, et al. Pacemaker implantation associated myocardial microdamage: a randomised comparison between active and passive fixation leads. Sci Rep. 2018;8:4870.

16. Wang J, Liang Y, Wang W, Chen X, Bai J, Chen H, et al. Left bundle branch area pacing is superior to right ventricular septum pacing concerning depolarization-repolarization reserve. J Cardiovasc Electrophysiol. 2020:31:313-22.

17. Zhang J, Wang Z, Zu L, Cheng L, Su R, Wang X, et al. Simplifying physiological left bundle branch area pacing using a new ninepartition method. Can J Cardiol. 2020; [Epub ahead of print].

18. Jiang Z, Chang Q, Wu Y, Ji L, Zhou X, Shan Q. Typical BBB morphology and implantation depth of 3830 electrode predict QRS correction by left bundle branch area pacing. Pacing Clin Electrophysiol. 2020:43:110-7.

19. Feng XF, Zhang PP, Liu B, Zhao Y, Lu QF, Li YG. Permanent left bundle branch area pacing utilizing intracardiac echocardiogram. BMC Cardiovasc Disord. 2020;20:377.

20. Vijayaraman P, Subzposh FA, Naperkowski A, Panikkath R, John $\mathrm{K}$, Mascarenhas V, et al. Prospective evaluation of feasibility and electrophysiologic and echocardiographic characteristics of left bundle branch area pacing. Heart Rhythm. 2019:16:1774-82.

21. Zhang J, Wang Z, Cheng L, Zu L, Liang Z, Hang F, et al. Immediate clinical outcomes of left bundle branch area pacing vs conventional right ventricular pacing. Clin Cardiol. 2019;42:768-73. 